\title{
The Use of Barbed Suture for Wound Closure in Hip and Knee Arthroplasty
}

\author{
*Olga Solovyova and James Slover \\ Langone Orthopedic Hospital, USA
}

Received: October 11,2017; Published: October 16, 2017

*Corresponding author: Olga Solovyova, NYU Langone Orthopedic Hospital, 301 East 17th Street, Ste. 1402, New York, NY 10003, USA; Email: Olga.solovyova@nyumc.org

Abbreviations: TKA: Total Knee Arthroplasty; CG: Control Group; BSG: Barbed Suture Group; PDO: Polydioxanone

\section{Mini Review}

The increasing number of total joint arthroplasties done on an annual basis, now over one million, along with evolving reimbursement strategies, has helped drive the need for more efficient performance of these cases [1]. This increased the demand for efficiency has led to pursuit of faster, more effective, safer, and cheaper surgical strategies. Soft tissue management is an integral part of total joint arthroplasty and its outcomes. Poor soft tissue handling can lead to dehiscence, infection, and unsightly scars. One of the more recent techniques in wound closure is the use of running barbed suture. The presence of either uni or bidirectional barbs eliminates the need for knots, potentially decreasing the time needed for closure and the number of sutures required and eliminating the gaps between sutures in the layer being closed. This paper summarizes the existing literature pertaining to the use of these suture constructs in total joint arthroplasty.

The use of these sutures for closure of the arthrotomy fascia in total knee arthroplasty (TKA) was examined in arandomized controlled trial of 170 patients, performed by Malhotra et al.This included 90 patients in the control group (CG) and 80 in the barbed suture group (BSG). Vicryl and Ethibond (Ethicon Inc, Somerville, NJ) were used for arthrotomy closure in the CG and Quill (Surgical Specialties Corporation, Wyomissing, PA) in the BSG. Vicryl and staples were used in all patients for subcuticular and skin closure in both groups. Wound closure time was four minutes slower and $\$ 7.00$ more expensive in the CG. There were five needle stick injuries in the CG, compared to zero in the BSG, but there were ten barbed suture breakages and none in the CG. There were no differences in complications or clinical outcomes between the groups [2].

Campbell compared the rates of wound complications between 247 TKA wound skin closures performed with Vicryl and staples to 169 wounds closed with running V-Loc (Medtronic, Minneapolis,
MN) barbed suture. They found that $18(7.3 \%)$ of the traditional closures and 33 (19.5\%) of the barbed suture closures developed wound problems $(p<0.001)$. Additionally, the staple group had a lower rate of eschar formation, but a higher incidence of keloid formation [3]. This suggests that barbed sutures may be problematic for more superficial layers of a surgical closure. A randomized controlled trial performed by Chan compared barbed suture (Stratafix, Ethicon Inc) for both the arthrotomy and skin closure (55 TKAs) and with Vicryl for both (54 TKAs). The Vicryl closure group had higher rates of fluid extravasation upon full flexion. Closure was less expensive and faster in the BSG and there were fewer wound complications (twoin the barbed group vs nine in the traditional group, $\mathrm{p}=0.03$ ). There was no difference in the rates of needle stick injuries, suture breakage, or glove perforation [4].

Chawla compared four groups undergoing unicompartmental knee arthroplasty. These included a group closed with barbed suture for the arthrotomy (Quill) and skin closure (Quill Monoderm), Vicryl for the arthrotomy and Monocryl for the skin, Vicryl for the arthrotomy and staples for skin, and Vicryl for the arthrotomy and Quill Monodermfor the skin. They found that out of 839 UKAs, with a minimum follow up of 16 weeks, there were eight infections, all of which had the skin closure performed with barbed suture. All infections occurred in the first two months, again suggesting these sutures may be problematic for closure of the superficial layers [5].

Eickmann and Quane compared 90 TKA closures done with barbed suture for the arthrotomy and skin (Quill) with 88 closures using Vicryl for the arthrotomy and sub-cuticular closure with Monocryl for the skin. The closures in the barbed suture group (BSG) were 11.5 minutes (13.3\%) faster. There was no difference in complication rates or postoperative range of motion. A multicenter 
RCT published by Gilliland et al compared 191 TKAs closed with barbed suture for the arthrotomy and subcuticular closure (Quill and Quill Monoderm) and 203 TKAs with Ethibond for the arthrotomy and Monocryl for the sub dermal layer, with both groups using staples for skin closure. Closure time was faster and cost was lower in the BSG, but there was a higher rate of suture breakage. There were no differences in complications or postoperative functional knee scores [6].

Maheshwari performed a retrospective review of 140 TKAs closed with one interrupted Ethibond followed by Quill for the arthrotomy, Quill for the sub-cuticular layer, and Monocryl for the skin with 193 TKAs which were closed with Ethibond and Vicryl for the arthrotomy, Vicryl for the subcuticular layer, and Ethilon for the skin. There were no differences in the closure or operative times. Closure was \$16 less expensive in the BSG. There were four wound complications in the CG and one in the BSG [7]. In an RCT conducted by Sah, fifty patients undergoing bilateral same day TKAs were randomizes to have one knee in the CG and one knee in the BSG. The CG closure was performed using Vicryl for the arthrotomy layer and Monocryl for the sub-cuticular and skin layers. The BSG closure used Quill for the arthrotomy and subcuticular layers and Quill Monoderm for skin. Wound closure with barbed suture was 4.7 minutes faster. There were five premature disengagements and three suture breakages in the CG and none in the BSG. There were no needle stick injuries and no differences in postoperative ROM or outcomes scores [8].

Smith et al performed an RCT comparing 18 total hip and knee arthroplasties closed with barbed suture and 16 arthroplasties in the control group. CG was closed using Ethibond, Vicryl, and Monocryl. The BSG used Quill and Quill Monoderm. Surgical time was 9.7 minutes faster in the BSG but $\$ 92$ more expensive. However, when taking into account the cost of operating room time, the authors found a savings of $\$ 550$ per arthroplasty in the BSG. There was no difference in wound complication rates in this cohort, nor in a retrospective review of 18 more controls and 80 more barbed suture closures. However, there was a trend of higher wound complications in the BSG, ten in 98 patients $(10.2 \%)$, as compared to two in 36 patients (5.6\%) in the CG [9].

Another RCT by Ting et al with 31 TJAs in the BSG and 29 patients in the CG. BSG used barbed polydioxanone (PDO) suture for all the layers, while in the CG, Vicryl and Monocryl were used. Closure was 4.2 minutes slower with more sutures used in the CG. There was no difference in complication rates. While the barbed sutures themselves were more expensive, when taking OR time into account, there was a cost savings of $\$ 615$ for THAs and $\$ 365$ for TKAs [10]. Due to concern for potential risk of glove perforation by the barbs on the sutures, Schwarzkopf et al. used electro conductivity testing to evaluate gloves which were used during closure of TJAs with and without barbed suture. Theydetermined that the rates of glove perforation were similar in cases which used barbed suture $(17$ of $122,13.9 \%$ ) and those that did not ( 9 of 50 , 18\%) [11].

Two studies evaluated bacterial adherence to barbed suture as compared to plain monofilament and braided suture. In an in vitro model, five suture types (Vicryl, Vicryl Plus, PDS, PDS Plus, and Quill) were tested as to the amount of bacterial adherence. Quill demonstrated the least and Vicryl plus the most [12]. An in vivo contaminated wound mouse model demonstrated that barbed monofilament and plain monofilament sutures were comparable. Braided suture performed inferiorly compared to the two other suture types in the amount of bacterial adherence, biofilm formation, and tissue reactivity [13].

\section{Summary}

In summary, several studies demonstrate that barbed suture is both time and cost saving in the operating room for closure of hip and knee arthroplasty wounds. There does not appear to be a difference in complication rates when barbed suture is used for the arthrotomy closure. However, several papers demonstrated increased wound complication rates when barbed suture was used for the subcuticular or skin closure, suggesting this may not be the best suture type for this superficial layer. No study demonstrated increased risk to the surgeon with the use of barbed suture, and bacterial adherence was not increased in the two studies evaluating this, suggesting it may be appropriate to use it in infected cases. Further studies are needed to determine the optimal closure technique for all patients, but barbed suturemay be optimal for use in total joint replacement patients.

\section{References}

1. Steiner C, AR Barrett M, Weiss A (2012) HCUP Projections: Mobility/ Orthopedic Procedures 2003 to 2012. HCUP Projections Report, USA.

2. Malhotra R, Jain V, Kumar V, Gautam D (2017) Evaluation of running knotless barbed suture for capsular closure in primary total knee arthroplasty for osteoarthritis-a prospective randomized study. Int Orthop, India.

3. Campbell AL, Patrick DA, Liabaud B, Geller JA (2014) Superficial wound closure complications with barbed sutures following knee arthroplasty. J Arthroplasty 29(5): 966-369.

4. Chan VWK, Chan PK, Chiu KY, Yan CH, Ng FY (2017) Does Barbed Suture Lower Cost and Improve Outcome in Total Knee Arthroplasty? A Randomized Controlled Trial. J Arthroplasty 32(5): 1474-1477.

5. Chawla H, Van der List JP, Fein NB, Henry MW, Pearle AD (2016) Barbed Suture Is Associated With Increased Risk of Wound Infection After Unicompartmental Knee Arthroplasty. J Arthroplasty 31(7): 1561-1567.

6. Eickmann T, E Quane (2010) Total knee arthroplasty closure with barbed sutures. J Knee Surg 23(3): 163-167.

7. Maheshwari AV, Wong A, Burko I, Mont MA, Rasquinha VJ (2015) Barbed sutures in total knee arthroplasty: are these safe, efficacious, and costeffective? J Knee Surg 28(2): 151-156.

8. Sah AP (2015) Is There an Advantage to Knotless Barbed Suture in TKA Wound Closure? A Randomized Trial in Simultaneous Bilateral TKAs. Clin Orthop Relat Res 473(6): 2019-2027.

9. Smith EL, DiSegna ST, Shukla PY, Matzkin EG (2014) Barbed versus traditional sutures: closure time, cost, and wound related outcomes in total joint arthroplasty. J Arthroplasty 29(2): 283-287.

10. Ting NT, Moric MM, Della Valle CJ, Levine BR (2012) Use of knotless suture for closure of total hip and knee arthroplasties: a prospective, randomized clinical trial. J Arthroplasty 27(10): 1783-1788.

11. Schwarzkopf R (2012) Barbed sutures for arthroplasty closure--does it decrease the risk of glove perforation? Bull NYU Hosp Jt Dis 70(4): 250253. 
12. Fowler JR, Perkins TA, Buttaro BA, Truant AL (2013) Bacteria adhere less to barbed monofilament than braided sutures in a contaminated wound model. Clin Orthop Relat Res 471(2): 665-671.
13. Morris MR, Bergum C, Jackson N, Markel DC (2017) Decreased Bacterial Adherence, Biofilm Formation, and Tissue Reactivity of Barbed Monofilament Suture in an In Vivo Contaminated Wound Model. J Arthroplasty 32(4): 1272-1279.

$\begin{array}{ll}\text { BIOMEDICAL } & \text { Assets of Publishing with us } \\ \text { RESEARCHES } & \text { - Global archiving of articles } \\ & \text { - Immediate, unrestricted online access } \\ & \text { - Aigorous Peer Review Process } \\ & \end{array}$

